\title{
Prevalence and determinants of the metabolic syndrome among Tunisian adults: results of the Transition and Health Impact in North Africa (TAHINA) project
}

\author{
Hanen Belfki ${ }^{1}$, Samir Ben Ali ${ }^{1}$, Hajer Aounallah-Skhiri ${ }^{1}$, Pierre Traissac $^{2}$, \\ Souha Bougatef ${ }^{1}$, Bernard Maire ${ }^{2}$, Francis Delpeuch ${ }^{2}$, Noureddine Achour $^{1}$ and \\ Habiba Ben Romdhane ${ }^{1, *}$ \\ 'Laboratory of Epidemiology and Prevention of Cardiovascular Disease, Faculty of Medicine, 15 rue Diebel \\ Akdhar-La Rabta-1007 Bab Saâdoun, Tunis, Tunisia: ${ }^{2}$ IRD (Institut de Recherche pour le Développement), \\ UMR 204 NUTRIPASS, IRD-UM1-UM2, Montpellier, France
}

Submitted 25 May 2011: Final revision received 17 April 2012: Accepted 31 May 2012: First published online 13 August 2012

\begin{abstract}
Objective: To determine the prevalence of metabolic syndrome (MetS) and its components and to evaluate the relationship between this diagnosis and cardiovascular risk factors, demographic and socio-economic variables.

Design: A cross-sectional study using a questionnaire including information on sociodemographic and CVD risk factors. Blood pressure, anthropometric indices, fasting glucose and lipid profile were measured. MetS was defined according to the criteria of the National Cholesterol Education Program, Adult Treatment Panel III.

Setting: The whole Tunisian territory; Transition and Health Impact in North Africa (TAHINA) project.

Subjects: A total of 4654 individuals (1840 men and 2814 women), aged 35 to 74 years, who participated in the Tunisian national survey.

Results: The overall prevalence of MetS was $30 \cdot 0 \%$, higher in women $(36 \cdot 1 \%)$ than in men $(20.6 \% ; P<0 \cdot 001)$. In both genders MetS prevalence increased significantly with age $(P<0 \cdot 001)$, but this increase was more important in women. Multiple regression analyses showed that the odds for MetS increased significantly with urban area for both men and women $(P<0.05$ and $P<0.001$, respectively). The multivariate models showed also that the odds for MetS increased significantly with increasing level of education and in those with a family history of CVD for men (both $P<0.05$ ) and after the menopausal transition for women $(P<0 \cdot 05)$.

Conclusions: The study highlights the MetS problem in a middle-income developing country. There is an urgent need for a comprehensive, integrated, population-based intervention programme to ameliorate the growing problem of MetS in Tunisians.
\end{abstract}

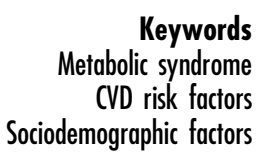

Metabolic syndrome (MetS) is a clustering of abdominal obesity, atherogenic dyslipidaemia, hypertension, insulin resistance and disturbed glucose metabolism that identifies individuals at increased risk for type 2 diabetes and progression of $\mathrm{CVD}^{(1)}$. MetS has become a global health problem and a substantial economic burden in both developing and developed countries ${ }^{(2,3)}$. Over the past two decades, Tunisia, a developing country in the Eastern Mediterranean Region, has undergone a sharp socioeconomic and demographic transition. Rapid changes in urbanization and socio-economic circumstances, related to the nutrition transition, are contributing to major epidemiological changes with the emergence of chronic diseases like obesity, diabetes, hypertension, CVD and other disorders ${ }^{(4-6)}$. The Tunisian epidemiological transition has been compounded by powerful environmental and behavioural changes. In particular, the adoption of new dietary habits and sedentary lifestyles, and the stress of urbanization and of working conditions, all may lead to increased prevalence of MetS and its risk factors ${ }^{(7)}$. Although modification of lifestyle-associated risk factors is known to be essential for preventing and managing MetS, there is currently insufficient information available on the prevalence of MetS and associated factors in North Africa and especially in Tunisia. In the Tunisian population, few studies on MetS have been conducted ${ }^{(8-10)}$. In addition, 
there is only one study conducted in the population of Greater Tunis which has investigated the implication of socio-economic status (SES) as assessed by education level in the prevalence of $\operatorname{MetS}^{(10)}$. An inverse relationship was found between education level and MetS only in women; however, this association is possibly confounded by factors other than age, which are strongly related to MetS. The present study was conducted in a nationally representative population to estimate the prevalence of MetS and its components among Tunisian adults and to evaluate the association of this syndrome with cardiovascular risk factors, demographic and socio-economic variables.

\section{Materials and methods}

\section{Sampling}

A large, nationally representative survey was conducted in the entire population of Tunisia. This survey was integrated into a collaborative project funded by the European Commission to study epidemiological Transition and Health Impact in North Africa (TAHINA). The survey was cross-sectional from April 2004 to September 2005 and the target population was adult men and women between the ages of 35 and 74 years. A nationally representative, stratified, two-tier cluster sample of households was selected according to the seven administrative regions of Tunisia. The sampling frame was derived by the Tunisian National Institute of Statistics from the database of the 2004 census of the population; in each stratum forty-seven census districts were selected, with a probability proportional to size in number of eligible households (i.e. featuring at least one 35-74-year-old household resident). At the second stage, twenty-five eligible households were sampled in each district; then for each household, all household residents from the targeted age range were to be included during the implementation of the field survey. The overall response rate was $79 \cdot 5 \%$, rates were generally lower in urban than in rural areas and in men than in women. The study protocol was carried out according to the Declaration of Helsinki and has been ethically approved by the Tunisian Ministry of Health and the Tunisian National Council of Statistics (visa $n^{\circ} 5 / 2005$ ). All participants gave their free informed consent, after being thoroughly informed of the purpose, requirements and procedures of the survey.

A total of 8007 people were enrolled. Of these, 3353 individuals who either had missing anthropometric measurements or did not fast properly prior to the blood test were excluded. The remaining 4654 participants (1840 men and 2814 women) constituted the sample used. The mean age of the 4654 individuals $(50 \cdot 2$ years for men, $50 \cdot 3$ years for women) was similar to that of the initial 8007 individuals recruited for the survey ( $49 \cdot 6$ years for men, 49.5 years for women). Excluded men had a higher prevalence of rural residents compared with included men $(P<0 \cdot 05)$. Excluded men had also a greater proportion of lower education levels $(P<0 \cdot 05)$. With respect to women, comparison between excluded and included groups showed no significant differences.

\section{Data collection}

Demographic and socio-economic information, smoking, menopausal status and taking of relevant medications (especially antidiabetic agents, antihypertensive agents and hormone replacement therapy) were obtained with a structured questionnaire pre-tested on a small group of non-participants. A family history of CVD was defined as having at least one parent or sibling diagnosed with diabetes, hypertension, stroke or CHD.

SES was assessed using the following measures obtained from the questionnaire: education level and occupation. Education was classified into four categories: level 0 , illiterate; level 1 , low-level education ( $\leq 6$ years of schooling); level 2, intermediate-level education (7-13 years of schooling); and level 3, higher education ( $\geq 14$ years of schooling). Occupation was classified into four categories: not working/retired; employee/ worker; intermediate grade (civil servants with $>6$ years of education); and upper grade (university graduates). Marital status was categorized as single, married, widowed or divorced. Concerning smoking status, a smoker was defined as a person who smoked any number of cigarettes regularly or who had any history of smoking in the past. A never smoker was defined as someone who had never smoked.

\section{Measurements}

Anthropometric measurements including weight and height were taken with the participant barefoot and wearing light clothing. Waist circumference (WC) was measured in the erect position at the middle between the tenth rib and the iliac crest. BMI $\left(\mathrm{kg} / \mathrm{m}^{2}\right)$ was calculated as the weight (in kilograms) divided by the square of the height (in metres). Systolic blood pressure (SBP) and diastolic blood pressure (DBP) were measured twice by a mercury sphygmomanometer in the sitting position after the participant had been quietly seated for more than $15 \mathrm{~min}$. The mean of the two measurements was used in the analyses. Blood samples were collected after a $12 \mathrm{~h}$ overnight fast. Fasting blood glucose (FBG) was determined by the glucose oxidase enzymatic method. Serum TAG was measured enzymatically after hydrolysis of glycerol and total cholesterol (TC) was measured by the cholesterol oxidase enzymatic method. All biochemical analyses were done using a Beckman reagent kit on a Beckman SYNCHRON CX7 analyser (Beckman Instruments, Inc., Fullerton, CA, USA).

\section{Definition of the metabolic syndrome}

According to the National Cholesterol Education Program, Adult Treatment Panel III (NCEP-ATPIII), MetS is 
defined as the presence of three or more of the following criteria: (i) $W C \geq 102 \mathrm{~cm}$ in men or $\geq 88 \mathrm{~cm}$ in women; (ii) $\mathrm{TAG} \geq 1.7 \mathrm{mmol} / \mathrm{l}$; (iii) $\mathrm{HDL}$ cholesterol (HDL-C) $<1.03 \mathrm{mmol} / 1$ in men or $<1.29 \mathrm{mmol} / 1$ in women; (iv) $\mathrm{SBP} \geq 130 \mathrm{mmHg}$ and/or $\mathrm{DBP} \geq 85 \mathrm{mmHg}$; and (v) $\mathrm{FBG} \geq 6 \cdot 1 \mathrm{mmol} / \mathrm{l}^{(11)}$. In the present study HDL-C was not determined because at the time of the survey the method used was the precipitation method, which is known for its large variability, and serum was not kept to measure it. We used TC instead of HDL-C as previously reported ${ }^{(9,12,13)}$. The cut-off point used was TC $\geq 5.2 \mathrm{mmol} / \mathrm{l}$ in men and women. Participants who reported taking hypertension or diabetes medications were considered to have elevated blood pressure (BP) or high FBG, respectively.

\section{Statistical analysis}

All statistical analyses were conducted using the SPSS statistical software package version $15 \cdot 0$ (SPSS Inc.). Continuous variables are presented as means and standard deviations, and categorical variables are presented as absolute and relative frequencies. Differences in baseline characteristics between groups were assessed using the independent $t$ test or the $\chi^{2}$ test, as appropriate. We describe the data stratified by gender because there was an interaction between gender and socio-economic factors in the association with MetS and in order to reduce the bias between the genders caused by the variation in response rate. We calculated the odds ratios together with their $95 \%$ approximate confidence intervals as estimators of the relative risk of MetS and its components for demographic characteristics and SES indicators of the study population. Univariate logistic regression analyses were used to determine crude odds ratios. To study the association of SES indicators and other factors with the prevalence of the MetS, we performed a multivariate logistic regression model with MetS as the dependent variable and with age, residence area, marital status, smoking, family history of CVD, education and occupation as the independent variables. The sampling design, i.e. stratification, clustering and sampling weights (accounting for differential probabilities of selection and also a post-stratification on gender, age and urban $v$. rural), was taken into account in all estimates and analyses using the specific SVY series of Stata commands. We added menopausal status to the model when analyses were done for women. The significance threshold was set at $P<0 \cdot 05$.

\section{Results}

The basic characteristics of the study population, stratified by gender, are listed in Table 1 . The overall prevalence of MetS was $30 \cdot 0 \%$, and was significantly higher in women than in men $(36 \cdot 1 \% v .20 \cdot 6 \%, P<0 \cdot 001)$. High WC, high $\mathrm{BP}$ and high $\mathrm{TC}$ were significantly more prevalent in women than in men $(P<0 \cdot 001, P<0.05$ and $P<0 \cdot 001$, respectively), while high FBG and high TAG were more prevalent in men $(P<0.05$ for both; Table 2$)$. The prevalence of MetS increased with age for both men and women $(P<0 \cdot 001$ for both), but the increase with age was more important in women. In the age group 35-44 years, $14.6 \%$ of men and $20.7 \%$ of women had MetS. In those over 64 years of age, the prevalence was $24 \cdot 1 \%$ and $51.5 \%$ for men and women, respectively (Fig. 1).

The OR for MetS and its components according to demographic and socio-economic indicators are shown in Tables 3 and 4 for men and women, respectively. In men, the odds for high WC $(\mathrm{OR}=1.31 ; P<0.05)$, high FBG $\quad(\mathrm{OR}=1.85 ; \quad P<0.001)$, high $\mathrm{TC} \quad(\mathrm{OR}=1.52$; $P<0 \cdot 05)$ and high TAG $(\mathrm{OR}=1.26 ; P<0 \cdot 05)$ were significantly higher in urban residents. Urban area was significantly associated with a higher odds for MetS, even after adjusting for covariates (OR $=1.51 ; \quad P<0 \cdot 05)$. Higher levels of education were significantly associated with higher odds for high WC $(P<0.05$ for low, intermediate and higher $v$. illiterate), high TC $(P<0.05$ for intermediate and higher $v$. illiterate) and high TAG $(P<0.05$ for low, intermediate and higher $v$. illiterate) and with lower odds for high BP $(P<0.05$ for low and intermediate $v$. illiterate). The odds for MetS increased linearly as education level increased, and this result was unchanged in multiple logistic regression analysis. Men with a family history of CVD showed higher odds for high WC $(P<0 \cdot 05)$, high BP $(P<0 \cdot 05)$ and high FBG $(P<0 \cdot 001)$. The odds for MetS was significantly higher in men with a family history of CVD before and after adjusting for confounding variables $(P<0.05$ for both). The odds increased for high TC $(P<0 \cdot 05)$ and high TAG $(P<0 \cdot 001)$ and decreased for high WC $(P<0 \cdot 05)$ and high BP $(P<0.05)$ in smokers compared with non-smokers. There was no significant effect of smoking, marital status and occupation on the risk of the MetS in univariate and multivariate logistic regression analyses (Table 3).

In women, urban residents showed higher odds for high WC (OR $=2 \cdot 06 ; P<0 \cdot 001)$, high FBG $(\mathrm{OR}=2 \cdot 04$; $P<0.001)$ and high $\mathrm{TC}(\mathrm{OR}=1.14 ; P<0 \cdot 001)$. The $\mathrm{OR}$ for MetS was significantly higher in urban residents before and after adjusting for risk factors $(P<0 \cdot 001$ for both). Postmenopausal women had higher odds for high WC (OR $=1 \cdot 26 ; P<0 \cdot 05)$, high $\mathrm{BP}(\mathrm{OR}=3 \cdot 25 ; P<0 \cdot 001)$, high FBG $(\mathrm{OR}=2 \cdot 51 ; P<0 \cdot 001)$ and high TC $(\mathrm{OR}=1 \cdot 88$; $P<0 \cdot 001)$ compared with premenopausal women. Postmenopausal stage was significantly associated with higher odds for MetS, even in the multivariate model $(\mathrm{OR}=1 \cdot 46$; $P<0 \cdot 05)$. The $\mathrm{OR}$ for high $\mathrm{BP}$ decreased linearly as the education level increased $(P<0.001$ for low, intermediate and higher $v$. illiterate). In contrast to what was found in men, in women the odds for MetS decreased significantly as the education level increased $(P<0.05$ for intermediate and higher $v$. illiterate), but this significance disappeared in the multivariate model. Women with 
Table 1 Baseline characteristics of the study participants and by gender: representative sample of Tunisian adults aged 35-74 years, Transition and Health Impact in North Africa (TAHINA) project, 2004-2005

\begin{tabular}{|c|c|c|c|c|c|c|}
\hline & \multicolumn{2}{|c|}{ All participants ( $n$ 4654) } & \multicolumn{2}{|c|}{ Men $(n 1840)$} & \multicolumn{2}{|c|}{ Women (n 2814) } \\
\hline & Mean & SD & Mean & SD & Mean & SD \\
\hline Age (years) & $50 \cdot 3$ & $9 \cdot 7$ & $50 \cdot 2$ & $9 \cdot 8$ & $50 \cdot 3$ & $9 \cdot 6$ \\
\hline WC $(\mathrm{cm})$ & $92 \cdot 4$ & $13 \cdot 0$ & $91 \cdot 9$ & $12 \cdot 5$ & $92 \cdot 6$ & $13 \cdot 2$ \\
\hline \multirow[t]{2}{*}{ BMI $\left(\mathrm{kg} / \mathrm{m}^{2}\right)$} & $27 \cdot 8$ & $8 \cdot 2$ & $25 \cdot 8$ & $4 \cdot 3$ & $29 \cdot 1$ & $9 \cdot 8$ \\
\hline & $n$ & $\%$ & $n$ & $\%$ & $n$ & $\%$ \\
\hline \multicolumn{7}{|l|}{ Residence area } \\
\hline Urban & 2748 & $59 \cdot 0$ & 1121 & $60 \cdot 9$ & 1627 & $57 \cdot 8$ \\
\hline Rural & 1906 & $41 \cdot 0$ & 719 & $39 \cdot 1$ & 1187 & $42 \cdot 2$ \\
\hline \multicolumn{7}{|l|}{ Marital status } \\
\hline Single & 117 & $2 \cdot 5$ & 45 & $2 \cdot 4$ & 72 & $2 \cdot 6$ \\
\hline Married & 4035 & $86 \cdot 7$ & 1733 & $94 \cdot 2$ & 2302 & $81 \cdot 8$ \\
\hline Widowed/divorced & 502 & $10 \cdot 8$ & 62 & $3 \cdot 4$ & 440 & $15 \cdot 6$ \\
\hline \multicolumn{7}{|l|}{ Smoking status } \\
\hline Smoker & 1301 & $28 \cdot 6$ & 1259 & $68 \cdot 9$ & 42 & $1 \cdot 5$ \\
\hline Never smoker & 3246 & $71 \cdot 4$ & 567 & $31 \cdot 1$ & 2679 & $98 \cdot 5$ \\
\hline \multicolumn{7}{|l|}{ Family history of CVD } \\
\hline No & 4504 & $96 \cdot 8$ & 1748 & $97 \cdot 8$ & 2720 & $97 \cdot 2$ \\
\hline Yes & 119 & $2 \cdot 6$ & 41 & $2 \cdot 2$ & 78 & $2 \cdot 8$ \\
\hline \multicolumn{7}{|l|}{ Menopausal status } \\
\hline Premenopausal & - & - & - & - & 1311 & $48 \cdot 9$ \\
\hline Postmenopausal & - & - & - & - & 1369 & $51 \cdot 1$ \\
\hline \multicolumn{7}{|l|}{ Education level } \\
\hline Illiterate & 2042 & $43 \cdot 9$ & 438 & $23 \cdot 9$ & 1604 & $57 \cdot 1$ \\
\hline Low & 1552 & $33 \cdot 3$ & 708 & $38 \cdot 7$ & 844 & $30 \cdot 1$ \\
\hline Intermediate & 805 & $17 \cdot 3$ & 510 & $27 \cdot 9$ & 295 & $10 \cdot 5$ \\
\hline Higher & 238 & $5 \cdot 1$ & 174 & $9 \cdot 5$ & 64 & $2 \cdot 3$ \\
\hline \multicolumn{7}{|l|}{ Occupation } \\
\hline Not working/retired & 2479 & $56 \cdot 6$ & 151 & $9 \cdot 1$ & 2328 & $85 \cdot 8$ \\
\hline Employee/worker & 1205 & $27 \cdot 5$ & 975 & $58 \cdot 5$ & 230 & $8 \cdot 5$ \\
\hline Intermediate & 202 & $4 \cdot 6$ & 136 & $8 \cdot 2$ & 66 & $2 \cdot 4$ \\
\hline Upper & 494 & $11 \cdot 3$ & 404 & $24 \cdot 2$ & 90 & $3 \cdot 3$ \\
\hline
\end{tabular}

WC, waist circumference.

Continuous variables are presented as means and standard deviations and were compared by the $t$ test. Categorical variables are presented as absolute and relative frequencies and were compared by the $\chi^{2}$ test.

Table 2 Prevalence of MetS and its components among the study participants and by gender: representative sample of Tunisian adults aged 35-74 years, Transition and Health Impact in North Africa (TAHINA) project, 2004-2005

\begin{tabular}{|c|c|c|c|c|c|c|}
\hline & \multicolumn{2}{|c|}{ All participants ( $n$ 4654) } & \multicolumn{2}{|c|}{ Men $(n$ 1840) } & \multicolumn{2}{|c|}{ Women ( $n$ 2814) } \\
\hline & $\%$ & $95 \% \mathrm{Cl}$ & $\%$ & $95 \% \mathrm{Cl}$ & $\%$ & $95 \% \mathrm{Cl}$ \\
\hline \multicolumn{7}{|l|}{ Component of MetS } \\
\hline High WC & $47 \cdot 7$ & $46 \cdot 3,49 \cdot 2$ & $20 \cdot 6$ & $18 \cdot 7,22 \cdot 4$ & $65 \cdot 5^{\star \star \star}$ & $63 \cdot 7,67 \cdot 2$ \\
\hline High BP & $49 \cdot 6$ & $48 \cdot 2,51 \cdot 0$ & $47 \cdot 6$ & $45 \cdot 3,49 \cdot 9$ & $50 \cdot 9^{\star}$ & $49 \cdot 0,52 \cdot 7$ \\
\hline High FBG & $14 \cdot 0$ & $13 \cdot 0,15 \cdot 0$ & $15 \cdot 2$ & $13 \cdot 6,16 \cdot 9$ & $13 \cdot 1^{*}$ & $11 \cdot 9,14 \cdot 4$ \\
\hline High TC & $19 \cdot 9$ & $18 \cdot 7,21 \cdot 0$ & $16 \cdot 1$ & $14 \cdot 4,17 \cdot 8$ & $22 \cdot 4^{\star \star \star}$ & $20 \cdot 8,23 \cdot 9$ \\
\hline High TAG & $54 \cdot 1$ & $52 \cdot 7,55 \cdot 6$ & $56 \cdot 6$ & $54 \cdot 4,58 \cdot 9$ & $52 \cdot 5^{\star}$ & $50 \cdot 6,54 \cdot 3$ \\
\hline MetS (NCEP-ATPIII definition) & $30 \cdot 0$ & $28 \cdot 6,31 \cdot 3$ & $20 \cdot 6$ & $18 \cdot 7,22 \cdot 4$ & $36 \cdot 1^{\star \star *}$ & $34 \cdot 3,37 \cdot 9$ \\
\hline
\end{tabular}

MetS, metabolic syndrome; WC, waist circumference; BP, blood pressure; FBG, fasting blood glucose; TC, total cholesterol; NCEP-ATPIII, National Cholesterol Education Program, Adult Treatment Panel III.

Values were significantly different from those in men: ${ }^{*} P<0.05,{ }^{* \star *} P<0.001$.

family history of CVD showed higher odds for high WC $(P<0 \cdot 05)$, high BP $(P<0 \cdot 05)$, high FBG $(P<0 \cdot 001)$ and high TC $(P<0 \cdot 05)$. The OR for MetS was higher in those with a family history of CVD $(P<0 \cdot 05)$, which was no longer significant after adjustment for covariates. Univariate and multiple logistic regression analyses showed no significant effect of smoking, marital status and occupation on the risk of MetS (Table 4).

\section{Discussion}

MetS is considered a problem of global proportions, affecting both developed and underdeveloped countries in a rapidly progressive way ${ }^{(3,14-17)}$. The prevalence in different countries, nevertheless, varies considerably. Such contrast is due to many factors. The most obvious is that different diagnostic criteria lead to different results, 


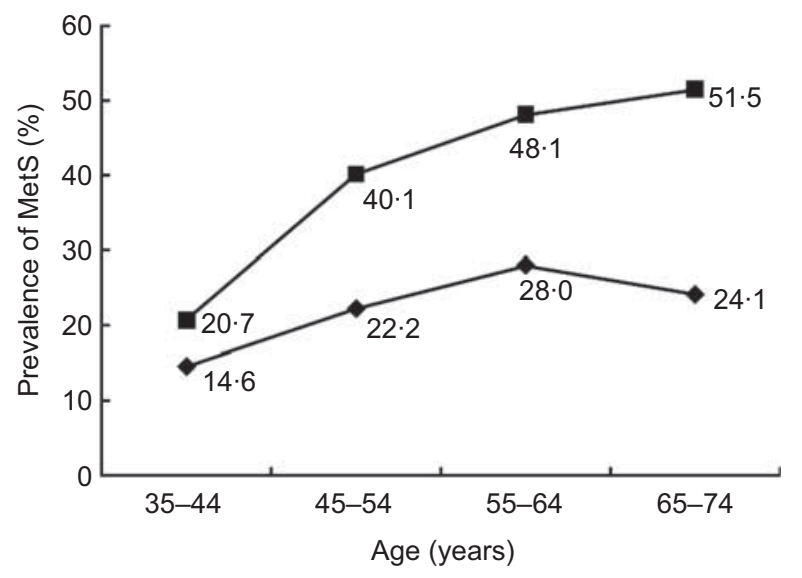

Fig. 1 Prevalence of metabolic syndrome (MetS) stratified by age group and gender (-৮-, men, $n$ 1840; - - - women, $n$ 2814): representative sample of Tunisian adults aged 35-74 years, Transition and Health Impact in North Africa (TAHINA) project, 2004-2005. MetS prevalence increased significantly with age for both genders: $P<0.001$

especially in specific subgroups ${ }^{(18-20)}$. Our data were based on the NCEP-ATPIII criteria, one of the most widely used in medical practice. In the present study, conducted in a large nationally representative sample of adults, $30.0 \%$ of the studied individuals had MetS. The prevalence of MetS was significantly higher in women $(36 \cdot 1 \%)$ than in men $(20 \cdot 6 \%)$. The MetS prevalence estimated in the current study is similar to that reported in a previous study carried out in a population of Greater Tunis, located in the north of Tunisia ${ }^{(10)}$. Several studies have reported an increased rate of MetS in women compared with men ${ }^{(8,10,21,22)}$. In the present study the higher MetS prevalence in women could be explained by the fact that central obesity was three times more frequent in women than in men $(65 \cdot 5 \% v$. 20.6\%, respectively). Central obesity has been shown to be an important predictor of CVD and diabetes ${ }^{(23,24)}$. The large WC in women may be explained by hormonal factors, postmenopausal weight gain and the general lack of participation in physical and sports activities among adult Tunisian women ${ }^{(25)}$. It has been suggested that multiple pregnancies may increase the risk of developing central obesity, insulin resistance and hypertension ${ }^{(26,27)}$, all components of MetS. We also found that age was an important factor in determining higher prevalence of MetS for both men and women, but the effect of age on MetS risk was more important in women. Similar results have been reported in many studies in other populations ${ }^{(16,28)}$.

With regard to the area of residence, urban area was independently associated with increased risk of MetS in both men and women. For both genders, urban residents showed higher OR for central obesity, diabetes and dyslipidaemia. Previous studies have documented higher MetS prevalence in the urban $v$. rural population ${ }^{(29-31)}$. Furthermore, these studies showed that rural dwellers had higher diet quality and physical activity than their urban counterparts. Urbanization often is associated with increased income and adoption of an unhealthy lifestyle, such as unhealthy food habits, with the transition from traditional rural diets (with a low glycaemic index and a higher fibre content) to a diet rich in salt, saturated fats and poor-quality carbohydrates (such as provided by fast foods) ${ }^{(32)}$. Furthermore, increased psychological stress, which is inevitable with urbanization, may be related to the development of $\mathrm{MetS}^{(33)}$.

In the present study we failed to detect an association between smoking and MetS, a result similar to that found in a Brazilian cohort ${ }^{(34)}$ but contrary to what has been reported in other studies ${ }^{(35,36)}$. We also studied the effect of family history of CVD on MetS. Results revealed that the risk of acquiring MetS was two- or threefold higher in men and women with a family history of CVD. After controlling for confounding factors, the result remained significant only for men. A positive family history of CVD has been reported to affect BP and serum glucose level ${ }^{(37,38)}$. Furthermore, individuals with a family history of CVD had an elevated risk for MetS ${ }^{(35)}$.

After menopause, oestrogen diminution is correlated with the appearance of symptoms and with changes in risks of disease that have an important impact on women's health $^{(39,40)}$. Postmenopausal status may explain in part the acceleration of CVD with ageing. Changes in the hormonal milieu at menopause contribute to the development of insulin resistance ${ }^{(41)}$. The present study showed that postmenopausal status was associated with increased odds for MetS, even after adjustment for confounding variables. This finding is in accordance with those reported by previous studies ${ }^{(42,43)}$.

Increasing interest is being taken in the relationship between SES and MetS. However, it has not been completely consistent between studies ${ }^{(28,34,35,44)}$. In Tunisia there is only one previous study conducted in the population of Greater Tunis investigating the relationship between education, as a socio-economic indicator, and MetS. An inverse relationship was found between this factor and MetS only in women ${ }^{(10)}$. However, in that study adjustment was done only for age. In the current study, we examined the association of education level and occupation with MetS adjusting for possible confounding factors. Our results revealed that men with elevated level of education had significantly greater odds of having central obesity and dyslipidaemia. In the same group, the odds of having MetS increased with increasing level of education, even in the multivariate model. In women, higher education levels were protective against hypertension. In contrast to what was observed in men, in women, education level was inversely related to MetS, but without significance after adjusting for potential confounding variables. Our findings are partially consistent with those reported in Korean adults ${ }^{(45)}$. With respect to occupation, no association was found between this variable and MetS 
Table 3 The odds for MetS and its components according to demographic and socio-economic indicators in men: representative sample of Tunisian adults aged 35-74 years, Transition and Health Impact in North Africa (TAHINA) project, 2004-2005

\begin{tabular}{|c|c|c|c|c|c|c|c|c|c|c|c|c|c|c|c|}
\hline & \multirow[b]{3}{*}{ n 1840} & \multicolumn{4}{|c|}{ MetS } & \multicolumn{10}{|c|}{ Component of MetS } \\
\hline & & \multicolumn{2}{|c|}{ Unadjustedt } & \multicolumn{2}{|c|}{ Multiple-adjusted } & \multicolumn{2}{|c|}{ High WC+ } & \multicolumn{2}{|c|}{ High BPt } & \multicolumn{2}{|c|}{ High FBG† } & \multicolumn{2}{|c|}{ High TC† } & \multicolumn{2}{|c|}{ High TAG† } \\
\hline & & OR & $95 \% \mathrm{Cl}$ & OR & $95 \% \mathrm{Cl}$ & OR & $95 \% \mathrm{Cl}$ & OR & $95 \% \mathrm{Cl}$ & OR & $95 \% \mathrm{Cl}$ & OR & $95 \% \mathrm{Cl}$ & OR & $95 \% \mathrm{Cl}$ \\
\hline \multicolumn{16}{|l|}{ Residence area } \\
\hline Rural§̧ & 115 & $1 \cdot 00$ & - & $1 \cdot 00$ & - & $1 \cdot 00$ & - & $1 \cdot 00$ & - & $1 \cdot 00$ & - & $1 \cdot 00$ & - & $1 \cdot 00$ & - \\
\hline Urban & 264 & $1 \cdot 61^{* \star *}$ & $1 \cdot 27,2 \cdot 06$ & $1 \cdot 51^{*}$ & $1 \cdot 14,1 \cdot 99$ & $1 \cdot 31^{*}$ & $1 \cdot 03,1 \cdot 66$ & $1 \cdot 10$ & $0.91,1 \cdot 33$ & $1 \cdot 85^{\star \star \star}$ & $1 \cdot 40,2 \cdot 45$ & $1 \cdot 52^{*}$ & $1 \cdot 16,1.99$ & $1 \cdot 26^{*}$ & $1.05,1.53$ \\
\hline \multicolumn{16}{|l|}{ Marital status } \\
\hline Single§ & 4 & $1 \cdot 00$ & - & $1 \cdot 00$ & - & $1 \cdot 00$ & - & $1 \cdot 00$ & - & $1 \cdot 00$ & - & $1 \cdot 00$ & - & $1 \cdot 00$ & - \\
\hline Married & 363 & $2 \cdot 71$ & $0.96,7 \cdot 63$ & $2 \cdot 96$ & $0 \cdot 89,9 \cdot 80$ & $2 \cdot 73$ & $0 \cdot 97,7 \cdot 68$ & $1 \cdot 65$ & $0.89,3.06$ & $1 \cdot 85$ & $0 \cdot 65,5 \cdot 20$ & $1 \cdot 56$ & $0.61,3.99$ & 1.52 & $0 \cdot 84,2 \cdot 76$ \\
\hline Widowed/divorced & 12 & $2 \cdot 46$ & $0 \cdot 73,8 \cdot 20$ & $1 \cdot 66$ & $0 \cdot 39,7 \cdot 06$ & 1.97 & $0 \cdot 57,6 \cdot 74$ & $2 \cdot 20$ & $1 \cdot 00,4 \cdot 84$ & $2 \cdot 46$ & $0 \cdot 73,8 \cdot 20$ & $1 \cdot 35$ & $0 \cdot 42,4 \cdot 36$ & $1 \cdot 14$ & $0.53,2.46$ \\
\hline \multicolumn{16}{|l|}{ Smoking status } \\
\hline Never smoker§ & 104 & $1 \cdot 00$ & - & 1.00 & - & 1.00 & - & $1 \cdot 00$ & - & 1.00 & - & $1 \cdot 00$ & - & $1 \cdot 00$ & - \\
\hline Smoker & 272 & $1 \cdot 22$ & $0.95,1.57$ & $1 \cdot 23$ & $0 \cdot 94,1 \cdot 62$ & $0 \cdot 68^{\star}$ & $0.54,0.87$ & $0 \cdot 74^{*}$ & $0.60,0.90$ & $1 \cdot 09$ & $0 \cdot 82,1 \cdot 44$ & $1 \cdot 41^{*}$ & $1 \cdot 06,1 \cdot 87$ & $1 \cdot 60^{\star \star *}$ & $1 \cdot 31,1.96$ \\
\hline \multicolumn{16}{|l|}{ Family history of CVD } \\
\hline None§ & 358 & $1 \cdot 00$ & - & $1 \cdot 00$ & - & $1 \cdot 00$ & - & 1.00 & - & $1 \cdot 00$ & - & $1 \cdot 00$ & - & $1 \cdot 00$ & - \\
\hline Yes & 17 & $2 \cdot 82^{*}$ & $1 \cdot 50,5 \cdot 30$ & $2 \cdot 21^{*}$ & $1 \cdot 11,4 \cdot 42$ & $2 \cdot 53^{*}$ & $1 \cdot 33,4 \cdot 79$ & $2 \cdot 40^{*}$ & $1 \cdot 23,4 \cdot 67$ & $2 \cdot 99^{\star \star *}$ & $1 \cdot 55,5 \cdot 79$ & 1.08 & $0 \cdot 47,2 \cdot 46$ & $1 \cdot 47$ & $0 \cdot 77,2 \cdot 83$ \\
\hline \multicolumn{16}{|l|}{ Education level } \\
\hline Illiterate§ & 76 & $1 \cdot 00$ & - & $1 \cdot 00$ & - & $1 \cdot 00$ & - & $1 \cdot 00$ & - & $1 \cdot 00$ & - & $1 \cdot 00$ & - & $1 \cdot 00$ & - \\
\hline Low & 135 & $1 \cdot 12$ & $0 \cdot 82,1 \cdot 53$ & $1 \cdot 65^{\star}$ & $1 \cdot 13,2 \cdot 41$ & $1 \cdot 40^{*}$ & $1 \cdot 01,1 \cdot 92$ & $0.67^{*}$ & $0.53,0.86$ & $0 \cdot 84$ & $0 \cdot 60,1 \cdot 19$ & $1 \cdot 14$ & $0 \cdot 80,1 \cdot 61$ & $1 \cdot 32^{\star}$ & $1.04,1.68$ \\
\hline Intermediate & 122 & $1 \cdot 49^{*}$ & $1 \cdot 08,2 \cdot 06$ & $2 \cdot 18^{\star \star \star}$ & $1 \cdot 43,3 \cdot 32$ & $1 \cdot 59^{*}$ & $1 \cdot 14,2 \cdot 22$ & $0 \cdot 76^{\star}$ & $0.59,0.99$ & $1 \cdot 23$ & $0 \cdot 86,1 \cdot 74$ & $1 \cdot 55^{\star}$ & $1 \cdot 09,2 \cdot 21$ & $1 \cdot 35^{*}$ & $1 \cdot 04,1 \cdot 75$ \\
\hline Higher & 44 & $1 \cdot 61^{*}$ & $1 \cdot 05,2 \cdot 45$ & $2 \cdot 24^{\star}$ & $1 \cdot 29,3 \cdot 89$ & $2 \cdot 29^{*}$ & $1 \cdot 51,3 \cdot 48$ & $0 \cdot 76$ & $0.53,1.08$ & $1 \cdot 39$ & $0 \cdot 88,2 \cdot 20$ & $1 \cdot 65^{\star}$ & $1 \cdot 04,2 \cdot 61$ & $1 \cdot 45^{\star}$ & $1 \cdot 01,2 \cdot 07$ \\
\hline \multicolumn{16}{|l|}{ Occupation } \\
\hline Upper§ & 103 & $1 \cdot 00$ & - & $1 \cdot 00$ & - & $1 \cdot 00$ & - & $1 \cdot 00$ & - & $1 \cdot 00$ & - & $1 \cdot 00$ & - & $1 \cdot 00$ & - \\
\hline Intermediate & 26 & 0.69 & $0 \cdot 42,1 \cdot 11$ & $0.55^{\star}$ & $0.33,0.91$ & 0.75 & $0.46,1.23$ & 0.93 & $0.63,1.37$ & $0 \cdot 84$ & $0.50,1 \cdot 42$ & 1.02 & $0.61,1.68$ & $1 \cdot 17$ & $0.78,1.74$ \\
\hline Employee/worker & 185 & 0.68 & $0.52,0.90$ & $0 \cdot 76$ & $0.56,1 \cdot 03$ & 0.79 & $0 \cdot 60,1 \cdot 05$ & $0 \cdot 86$ & $0 \cdot 68,1 \cdot 08$ & 0.65 & $0.47,0.89$ & $0 \cdot 77$ & $0.56,1.05$ & 0.97 & $0.77,1.23$ \\
\hline Not working/retired & 29 & 0.69 & $0 \cdot 43,1 \cdot 10$ & $0 \cdot 66$ & $0.40,1.09$ & $0 \cdot 86$ & $0.54,1.36$ & $1 \cdot 59^{*}$ & $1 \cdot 08,2 \cdot 32$ & 0.74 & $0 \cdot 44,1 \cdot 25$ & 0.94 & $0.57,1.54$ & $0.59^{*}$ & $0.40,0.86$ \\
\hline
\end{tabular}

MetS, metabolic syndrome; WC, waist circumference; BP, blood pressure; FBG, fasting blood glucose; TC, total cholesterol.

Odds ratios were significant compared with the referent category: ${ }^{*} P<0.05,{ }^{* \star *} P<0.001$

¥Multivariate logistic regression analysis including MetS as the dependent variable and age, residence area, marital status, smoking, family history of CVD, educational level and occupation as independent variables. §Referent category 
Table 4 The odds for MetS and its components according to demographic and socio-economic indicators in women: representative sample of Tunisian adults aged 35-74 years, Transition and Health Impact in North Africa (TAHINA) project, 2004-2005

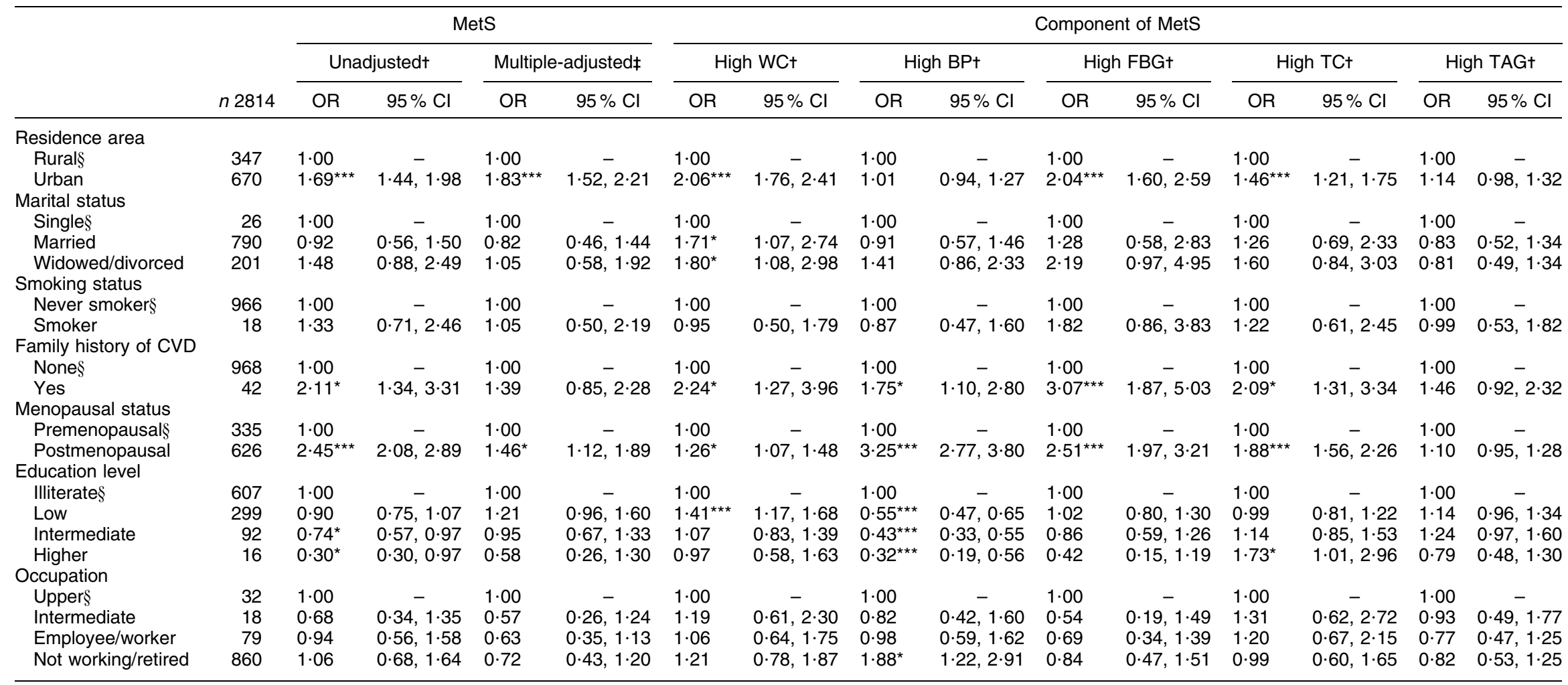

MetS, metabolic syndrome; WC, waist circumference; BP, blood pressure; FBG, fasting blood glucose; TC, total cholesterol.

Odds ratios were significant compared with the referent category: ${ }^{*} P<0.05,{ }^{* * *} P<0.001$

¥Multivariate logistic regression analysis including MetS as the dependent variable and age, residence area, marital status, smoking, family history of CVD, educational level and occupation as independent variables. $\S$ Referent category. 
in both genders. There is some explanation for the gender difference in the relationship between education and MetS. Lower educational level is related to high morbidity and mortality from CVD, a relationship that is clearer among women than men ${ }^{(46)}$. Women with low levels of education have additional psychosocial risks (including high depressive symptoms, income under the poverty threshold, unemployment and single parenting) compared with men with low education ${ }^{(47)}$. However, highly educated women are provided with greater levels of social, psychological and economic support ${ }^{(48)}$. Furthermore, women with higher SES generally are more aware of the importance of health and fitness, and therefore tend to consume a healthy diet and regularly practise sports and exercise. In contrast, men of higher SES lead a more sedentary lifestyle and have a greater opportunity for smoking and consuming alcohol and rich foods ${ }^{(10)}$. Future research focused on identifying mechanisms responsible for gender differences in the relationship between SES and MetS will provide better knowledge of the potential pathways.

Our study has strengths and limitations. The strengths include: the large sample consisting of both urban and rural populations; a sound representation of the national population; and detailed information on potential confounding factors. As a cross-sectional study, the present analysis is, however, limited in its ability to elucidate a causal relationship. The response rate was also less than desired as only $79.5 \%$ of contacted participants and $58.0 \%$ of targeted participants were included in the study. This introduces the possibility of selection bias and therefore limits the generalizability of the findings. Participants with missing values of the risk factors necessary for a diagnosis of MetS were excluded from the analysis to ensure that all analyses were done on the same data. There were no differences in baseline characteristics between those excluded and those included in the analysis. It is therefore unlikely that any bias resulted from the exclusion of persons with missing data. Another limitation was the absence of the measurement of HDL-C as explained above. Information about diet and physical activity was not included in the study.

\section{Conclusion}

The prevalence of MetS is high in both men and women in Tunisia. Mets is not only a concern for the urban population, but is also a matter of debate in rural areas. To reduce the risk of Mets, a multidimensional effort by individuals, society and the national health programme is necessary. Future prospective studies are also needed to clarify other potential risks for MetS.

\section{Acknowledgements}

The study was financed by the European Commission (ICA3-CT-2002-1001) and supported by a grant from the
Ministry of Public Health of Tunisia. The authors declare that they have no competing interests. H.B. conceived the protocol, managed data collection and drafted the manuscript. S.B.A. researched the literature, conducted statistical analyses, contributed to interpretation of the results and to writing the manuscript. H.A.-S. and S.B. contributed to data collection management. P.T., B.M., F.D., N.A. and H.B.R. contributed to the study design and revision of the manuscript. All authors reviewed and edited the manuscript and approved the final version of the manuscript. The authors extend appreciation to all of the dedicated field workers and those who helped facilitate the field work in each survey site.

\section{References}

1. Grundy SM, Cleeman JI, Daniels SR et al. (2005) Diagnosis and management of the metabolic syndrome: an American Heart Association/National Heart, Lung, and Blood Institute Scientific Statement. Circulation 112, 2735-2752.

2. Steinberger J, Daniels SR, Eckel RH et al. (2009) Progress and challenges in metabolic syndrome in children and adolescents: a scientific statement from the American Heart Association Atherosclerosis, Hypertension, and Obesity in the Young Committee of the Council on Cardiovascular Disease in the Young; Council on Cardiovascular Nursing; and Council on Nutrition, Physical Activity, and Metabolism. Circulation 119, 628-647.

3. Gu D, Reynolds K, Wu X et al. (2005) Prevalence of the metabolic syndrome and overweight among adults in China. Lancet 365, 1398-1405.

4. Popkin BM (2001) The nutrition transition and obesity in the developing world. $J$ Nutr 131, issue 3, 871S-873S.

5. Maire B, Lioret S, Gartner A et al. (2002) Nutritional transition and non-communicable diet-related chronic diseases in developing countries. Sante 12, 45-55.

6. Ntandou G, Delisle H, Agueh V et al. (2008) Physical activity and socioeconomic status explain rural-urban differences in obesity: a cross-sectional study in Benin (West Africa). Ecol Food Nutr 47, 313-337.

7. Ben Romdhane H, Habib H, Ali B et al. (2005) Epidemiological transition and health impact of cardiovascular disease in Tunisia. Tun Med 83, 2486-2497.

8. Harzallah F, Alberti H \& Ben Khalifa F (2006) The metabolic syndrome in an Arab population: a first look at the new International Diabetes Federation criteria. Diabet Med 23, 441-444.

9. Bouguerra R, Ben Salem L, Alberti H et al. (2006) Prevalence of metabolic abnormalities in the Tunisian adults: a population based study. Diabetes Metab 32, 215-221.

10. Allal-Elasmi M, Haj Taieb S, Hsairi M et al. (2010) The metabolic syndrome: prevalence, main characteristics and association with socio-economic status in adults living in Great Tunis. Diabetes Metab 36, 204-208.

11. Expert Panel on Detection, Evaluation, and Treatment of High Blood Cholesterol in Adults (2001) Executive Summary of the Third Report of the National Cholesterol Education Program (NCEP) Expert Panel on Detection, Evaluation, and Treatment of High Blood Cholesterol in Adults (Adult Treatment Panel III). JAMA 285, 2486-2497.

12. Rguibi M \& Belahsen R (2004) Metabolic syndrome among Moroccan Sahraoui adult women. Am J Hum Biol 16, 598-601.

13. Fezeu L, Balkau B, Kengne AP et al. (2007) Metabolic syndrome in a sub-Saharan African setting: central obesity may be the key determinant. Atherosclerosis 193, 70-76. 
14. Ford ES (2004) Prevalence of the metabolic syndrome in US populations. Endocrinol Metab Clin North Am 33, 333-350.

15. Kraja AT, Rao DC, Weder AB et al. (2005) An evaluation of the metabolic syndrome in a large multi-ethnic study: the family blood pressure program. Nutr Metab (Lond) 2, 17.

16. Miccoli R, Bianchi C, Odoguardi L et al. (2005) Prevalence of the metabolic syndrome among Italian adults according to ATP III definition. Nutr Metab Cardiovasc Dis 15 , 250-254.

17. Oh JY, Hong YS, Sung YA et al. (2004) Prevalence and factor analysis of metabolic syndrome in an urban Korean population. Diabetes Care 27, 2027-2032.

18. Alberti KG, Zimmet P \& Shaw J (2006) Metabolic syndrome - a new worldwide definition. A consensus statement from the International Diabetes Federation. Diabet Med 23 , 469-480.

19. Ford ES \& Giles WH (2003) A comparison of the prevalence of the metabolic syndrome using two proposed definitions. Diabetes Care 26, 575-581.

20. Cheung BM, Ong KL, Man YB et al. (2006) Prevalence of the metabolic syndrome in the United States National Health and Nutrition Examination Survey 1999-2002 according to different defining criteria. J Clin Hypertens (Greenwich) 8, 562-570.

21. Velásquez-Meléndez G, Gazzinelli A, Côrrea-Oliveira R et al. (2007) Prevalence of metabolic syndrome in a rural area of Brazil. Sao Paulo Med J 125, 155-162.

22. Jaber LA, Brown MB, Hammad A et al. (2004) The prevalence of the metabolic syndrome among Arab Americans. Diabetes Care 27, 234-238.

23. Yusuf S, Hawken S, Ounpuu S et al. (2005) Obesity and the risk of myocardial infarction in 27,000 participants from 52 countries: a case-control study. Lancet 366, 1640-1649.

24. Wang Y, Rimm EB, Stampfer MJ et al. (2005) Comparison of abdominal adiposity and overall obesity in predicting risk of type 2 diabetes among men. Am J Clin Nutr 81, 555-563.

25. Ghannem H (2006) The challenge of preventing cardiovascular disease in Tunisia. Prev Chronic Dis 3, A13.

26. Lasky D, Becerra E, Boto W et al. (2002) Obesity and gender differences in the risk of type 2 diabetes mellitus in Uganda. Nutrition 18, 417-421.

27. Catalano PM (2003) Obesity and pregnancy - the propagation of a vicious cycle? J Clin Endocrinol Metab 88, 3505-3506.

28. Park HS, Oh SW, Cho S et al. (2004) The metabolic syndrome and associated lifestyle factors among South Korean adults. Int J Epidemiol 33, 328-336.

29. Weng X, Liu Y, Ma J et al. (2007) An urban-rural comparison of the prevalence of the metabolic syndrome in Eastern China. Public Health Nutr 10, 131-136.

30. Das M, Pal S \& Ghosh A (2008) Rural urban differences of cardiovascular disease risk factors in adult Asian Indians. Am J Hum Biol 20, 440-445.

31. Ntandou G, Delisle H, Agueh V et al. (2009) Abdominal obesity explains the positive rural-urban gradient in the prevalence of the metabolic syndrome in Benin, West Africa. Nutr Res 29, 180-189.
32. Mittal BV \& Singh AK (2010) Hypertension in the developing world: challenges and opportunities. $\mathrm{Am} \mathrm{J}$ Kidney Dis 55, 590-598.

33. Tamashiro KL (2011) Metabolic syndrome: links to social stress and socioeconomic status. Ann N Y Acad Sci 1231, $46-55$.

34. da Silveira VM, Horta BL, Gigante DP et al. (2010) Metabolic syndrome in the 1982 Pelotas cohort: effect of contemporary lifestyle and socioeconomic status. Arq Bras Endocrinol Metabol 54, 390-397.

35. Lee WY, Jung CH, Park JS et al. (2005) Effects of smoking, alcohol, exercise, education, and family history on the metabolic syndrome as defined by the ATP III. Diabetes Res Clin Pract 67, 70-77.

36. Steemburgo T, Dall'Alba V, Grosss JL et al. (2007) Dietary factors and metabolic syndrome. Arq Bras Endocrinol Metabol 51, 1425-1433.

37. Válek J \& Vlasáková Z (1997) The metabolic syndrome, its heredity, methods of detection and clinical significance. Vnitr Lek 43, 566-573.

38. Hunt KJ, Heiss G, Sholinsky PD et al. (2000) Familial history of metabolic disorders and the multiple metabolic syndrome: the NHLBI family heart study. Genet Epidemiol 19, 395-409.

39. Silva TCBF, Barrett-Connor E, Ramires JAF et al. (2008) Obesity, estrone, and coronary artery disease in postmenopausal women. Maturitas 59, 242-248.

40. Al-Azzawi F \& Palacios S (2009) Hormonal changes during menopause. Maturitas 63, 135-137.

41. Kalish GM, Barrett-Connor E, Laughlin GA et al. (2003) Association of endogenous sex hormones and insulin resistance among postmenopausal women: results from the Postmenopausal Estrogen/Progestin Intervention Trial. $J$ Clin Endocrinol Metab 88, 1646-1652.

42. Eshtiaghi R, Esteghamati A \& Nakhjavani M (2010) Menopause is an independent predictor of metabolic syndrome in Iranian women. Maturitas 65, 262-266.

43. Janssen I, Powell LH, Crawford S et al. (2008) Menopause and the metabolic syndrome: the Study of Women's Health Across the Nation. Arch Intern Med 168, 1568-1575.

44. Loucks EB, Rehkopf DH, Thurston RC et al. (2007) Socioeconomic disparities in metabolic syndrome differ by gender: evidence from NHANES III. Ann Epidemiol 17, 9-26.

45. Park MJ, Yun KE, Lee GE et al. (2007) A cross-sectional study of socioeconomic status and the metabolic syndrome in Korean adults. Ann Epidemiol 17, 320-326.

46. Heller RF, Williams H \& Sittampalam Y (1984) Social class and ischemic heart disease: use of the male:female ratio to identify possible occupational hazards. J Epidemiol Community Health 38, 198-202.

47. Thurston RC, Kubzansky LD, Kawachi I et al. (2005) Is the association between socioeconomic position and coronary heart disease stronger in women than in men? $\mathrm{Am}$ J Epidemiol 162, 57-65.

48. Winkleby MA, Jatulis DE, Frank E et al. (1992) Socioeconomic status and health: how education, income, and occupation contribute to risk factors for cardiovascular disease. Am J Public Health 82, 816-820. 